\title{
Adaptação da Escala de Uso Compulsivo da Internet (CIUS) para o Brasil
}

Adaptation of the Compulsive Internet Use Scale (CIUS) to Brazil

Adaptación de la Escala de Uso Compulsivo de Internet (CIUS) para Brasil

Emerson Diógenes de Medeiros - emersondiogenes@gmail.com Universidade Federal do Delta do Parnaíba, Parnaíba-PI, Brasil. ORCID: https://orcid.org/0000-0002-1407-3433

Hemerson Fillipy Silva Sales - fillipysilva@hotmail.com Universidade Federal da Paraíba, João Pessoa-PB, Brasil. ORCID: https://orcid.org/0000-0001-5603-0960

Bruna de Jesus Lopes - bruna_lopespsi@hotmail.com

Faculdade Maurício de Nassau, UNINASSAU, Parnaíba-PI, Brasil. ORCID: https://orcid.org/0000-0003-0382-2213

Anderson Mesquita do Nascimento - andermnascimento@gmail.com Universidade do Estado de Minas Gerais, Ituiutaba-MG, Brasil. ORCID: https://orcid.org/0000-0001-5093-8353

Tailson Evangelista Mariano - tailsonmariano@hotmail.com Universidade Federal da Paraíba, João Pessoa-PB, Brasil. ORCID: https://orcid.org/0000-0001-6716-0250

Paulo Gregório Nascimento da Silva - silvapgn@gmail.com Universidade Federal da Paraíba, João Pessoa-PB, Brasil. 
ORCID: https://orcid.org/0000-0002-2878-309X

\begin{abstract}
Resumo
O presente estudo objetivou adaptar a Escala de Uso Compulsivo da Internet (CIUS) para o Brasil verificando seus parâmetros de validade e precisão. Para isso contou-se com uma amostra de 201 usuários de internet encontrados em redes sociais, sendo a maioria do Piaú (84\%), do sexo feminino $(55,7 \%)$ e com idades variando entre 13 e 45 anos $(M=22,50 ; D P=4,55)$. A princípio foi verificado o poder discriminativo dos itens, onde todos se apresentaram satisfatórios. Posteriormente para verificar a validade do instrumento, utilizou-se de uma Análise dos Componentes Principais, que apontou uma estrutura unidimensional; e uma análise de Regressão Bivariada que confirmou sua validade preditiva. Por fim verificou-se a precisão através do coeficiente alfa de Cronbach, obtendo-se um alfa $\alpha=0,91$. Conclui-se com esses resultados, que a CIUS possui bons parâmetros psicométricos, podendo assim, ser usada em estudos futuros para identificar usuários compulsivos de internet.
\end{abstract}

Palavras-chave: Uso compulsivo da internet; Validade; Precisão.

\begin{abstract}
This study aimed to adapt Compulsive Internet Use Scale (CIUS) to Brazil, verifying their validity and accuracy. To this end, the sample consisted of 201 internet users found on social networks, most of Piaui (84\%), female (55.7\%) and aged between 13 and 45 years $(M=22.50, S D=4.55)$. Firstly, was verified the discriminative power of items, where everyone showed satisfactory. Subsequently to check the validity of the instrument, we used a Principal Component Analysis, which indicated a unidimensional structure, and a Bivariate Regression analysis confirmed that its predictive validity. Finally, it was found consistency by Cronbach's alpha coefficient, $\alpha=0.91$. The findings show that the CIUS has good psychometric parameters and can therefore be used in future studies to identify compulsive internet users.
\end{abstract}

Keywords: Compulsive Internet use; Validity, Accuracy.

\title{
Resumen
}

Este estudio tenía por objeto adaptar la Escala de Uso Compulsivo de Internet (CIUS) para el Brasil, comprobando sus parámetros de validez y precisión. Para ello se incluyó una muestra de 201 usuarios de Internet encontrados en las redes sociales, la mayoría de ellos de Piaui (84\%), mujeres (55, 7\%) y de entre 13 y 45 años ( $M=22,50$; $S D=4,55)$. Al principio, se verificó el poder de discriminación de los artículos, donde todos eran satisfactorios. Posteriormente, para verificar la validez del instrumento, se utilizó un Análisis de Componentes Principales, que señaló una estructura unidimensional; y un Análisis de Regresión Bivariada que confirmó su validez predictiva. Finalmente, la precisión fue verificada a través del coeficiente alfa de Cronbach, obteniendo un alfa $\alpha=0,91$. Estos resultados muestran que el CIUS tiene buenos parámetros psicométricos y, por lo tanto, puede utilizarse en futuros estudios para identificar a los usuarios compulsivos de Internet.

Palabras clave: Uso compulsivo de Internet; Validez; Precisión.

\section{Introdução}


Com o advento de novas tecnologias, muitos dos meios criados pelo homem para facilitar sua vida, e que se mostram muito úteis e benéficos na atualidade, vem se tornando cada vez mais essenciais à vida cotidiana. No entanto, algumas dessas tecnologias quando utilizadas de forma exagerada podem deixar de ser benéficas e se tornarem prejudiciais para o indivíduo. A internet, assim como os jogos eletrônicos (Bean, Nielsen, van Rooij, \& Ferguson, 2017; Deleuze, Long, Liu, Maurage, \& Billieux, 2018) é um exemplo disso.

Utilizando-se de um ponto de vista comportamental, autores comparam (Meerkerk, Van Den Eijnden, Franken, \& Garretsen, 2010), o sistema homem - internet, a uma rede de caixas de Skinner adaptadas individualmente, onde os comportamentos dos seus usuários seriam reforçados através de mecanismos de condicionamento clássico e operante, em que através de reforços positivos intermitentes tais comportamentos seriam compostos de forma gradual, tornando-os cada vez mais qualificados para encontrar mais estímulos na internet que se adapte a eles; fazendo com que os mesmos a utilizem de uma forma abusiva.

Segundo Abreu, Karam, Góes e Spritzer, (2008), na literatura internacional, o uso abusivo da internet é definido de diferentes formas, sendo a ele atribuído terminologias diversas que variam em função das diferentes áreas de atuação dos profissionais que buscam compreendê-lo. Uma das primeiras e mais utilizada é a terminologia Internet Addiction, seguida pelo termo Problematic Internet Use, mas não se restringem a estes, podendo encontrar ainda Pathological Internet Use, Internet Addiction Disorder, Compulsive Internet Use, Computer Mediated Communications Addicts, Computer Junkies e Internet Dependency. Dessas terminologias as mais contestadas são aquelas que apontam a internet como um vício.

No presente estudo, levando em consideração que o tema não trata de um vício em internet geral e sim de uma mudança de comportamento referente ao aumento da frequência de certas atividades online, foi utilizada a terminologia uso compulsivo da internet (Meerkerk, Van Den Eijnden, Vermulst, \& Garretsen, 2009). O Uso Compulsivo da Internet (UCI) refere-se a um padrão de uso, caracterizado pelos principais elementos do comportamento compulsivo ou aditivo, aplicáveis a utilização da internet, como a perda de controle, a preocupação, o conflito, os sintomas de abstinência e o uso da internet como estratégia de enfrentamento (Meerkerk et al., 2009; Meerkerk et al., 2010). 
Segundo Meerkerk et al. (2009), como já foi mencionado, não existiria de fato o vício na internet em geral, mas sim o uso de forma problemática de algumas atividades online. Desse modo, para este autor o UCI abrangeria várias formas de comportamentos compulsivos ou viciantes, a exemplo do comportamento sexual na Internet (Studer, Marmet, Wicki, \& Gmel, 2019) ou mesmo o vício em jogos on-line (Anderson \& Bushman, 2018), sendo a internet, o meio pelos quais esses comportamentos seriam realizados ou facilitados.

A literatura sugere que o uso excessivo da Internet pode se tratar de um novo transtorno psiquiátrico (Abreu et al., 2008). No entanto, os estudos existentes na área são bastante heterogêneos, já que há uma variabilidade muito grande de nomenclatura na literatura, além da escassez de critérios diagnósticos e definições operacionais que possibilitem o estabelecimento de uma síndrome clinicamente identificável (Griffiths, 1997). Os critérios diagnósticos mais comuns, existentes para a identificação de problemas relacionados ao UCI ou o vício em internet, são baseados em critérios derivados daqueles utilizados pelo DSM-IV (APA, 1994) para diagnosticar dependência de substâncias e transtorno obsessivo-compulsivo (Meerkerk et al., 2009).

Entre os problemas psicológicos mais comumente relacionados ao UCI, podemos citar: a depressão (Rosenkranz, Müller, Dreier, Beutel, \& Wölfling, 2017), a impulsividade (Mottram \& Fleming, 2009), a timidez (Ebeling-Witte, Frank, \& Lester, 2007), cyberbullying (Sebastian et al., 2018), a ansiedade (Pontes, 2017), a ansiedade social (Van Zalk, 2016). e desempenho escolar (Rosenkranz et al., 2017). Além disso, também poder vir a causar problemas familiares (Wartberg, Kriston, Bröning, Kegel, \& Thomasius, 2017), ou mesmo menor confiança interpessoal (Rosenkranz et al., 2017).

Tendo em conta tais problemas, associados ao uso compulsivo da internet, diversos instrumentos foram propostos com o intuito de mensurar o uso nocivo da internet, através de aspectos diversos, sendo os mais comuns: o vício em internet (Korean Scale for Internet Addiction; Mak et al., 2017). No entanto, para o presente trabalho a Escala de Uso Compulsivo da Internet de Meerkerk et al. (2009), foi utilizada, pois reúne qualidades que a fazem promissora. Trata-se de uma escala unifatorial, construída a partir de uma análise dos critérios utilizados para dependência e transtorno obsessivocompulsivo descritos no DSM-IV. Esta escala vem sendo utilizada em estudos diversos (Meerkerk et al., 2010; Kerkhof, Finkenauer, \& Muusses, 2011) tendo sido inclusive, 
adaptada em outros contextos e línguas. Em um estudo de Lopez-Fernandez et al. (2019), quatro versões do CIUS (CIUS-14, CIUS-9, CIUS-7 e CIUS-5) forma testadas em oito idiomas (alemão, francês, inglês, finlandês, espanhol).

Atualmente, não existem muitos estudos sobre o UCI no Brasil. A pouca literatura existente se restringe ainda a pesquisas qualitativas (Abreu et al., 2008; Penteado, Santos, Araújo, \& Silva, 2011) não sendo encontrado, no material consultado, pesquisas de cunho quantitativo, e consequentemente instrumentos que identifiquem o UCI.

\section{Objetivos}

Justificando o objetivo principal deste estudo, ou seja, o de adaptar ao contexto nacional tal instrumento. Especificamente buscou-se conhecer evidências de seus parâmetros de validade e precisão.

\section{Método}

\section{Participantes}

Contou-se com uma amostra por conveniência (não probabilística) de 201 usuários de internet distribuídos em vários estados brasileiros, sendo a maior parte do Piauí (84\%); e com idades variando de 13 a 45 anos $(M=22,50 ; D P=4,55)$, a maioria do sexo feminino $55,7 \%$.

\section{Instrumento}

Fez-se uso de um formulário digitalizado subdividido em duas partes. $\mathrm{Na}$ primeira, os participantes tinham que responder a um questionário sócio demográfico e na segunda, a Escala do Uso Compulsivo da Internet (CIUS).

Questionário sócio demográfico - nessa parte do formulário, com o objetivo de caracterizar os participantes dessa pesquisa, foram utilizadas algumas perguntas referentes à idade e ao sexo dos respondentes; sendo incluída também uma pergunta descrita: “Aproximadamente, quantas horas por dia você gasta usando a internet?".

Escala de Uso Compulsivo da Internet (CIUS) - A Compulsive Internet Use Scale (CIUS) é um instrumento composto por 14 itens que descrevem comportamentos de uso compulsivo da internet como, a exemplo de "você continua a usar a Internet, apesar de sua intenção de parar?”. A mesma possui uma estrutura unidimensional, sendo que suas respostas são dadas em uma escala do tipo Likert de 5 pontos (que varia de $0=$ nunca a 4 
$=$ muito frequentemente), referentes a frequência que se emite os comportamentos descritos nos itens (Meerkerk et al., 1999). A mesma foi traduzida através da técnica de back translation, que consiste na tradução dos itens do idioma original (inglês) para o idioma da amostra pretendida (português do Brasil), e depois para o idioma original novamente. Após a tradução dos itens, foi realizada ainda uma análise semântica destes, com o intuito de verificar se eles eram compreensíveis (Pasquali, 2016). Participaram desta análise 12 usuários de internet, sendo que nenhum deles apresentou dificuldade em compreender o questionário.

\section{Procedimento}

Após a tradução, elaborou-se o formulário eletrônico contendo os instrumentos alvos deste estudo em um formulário de um site de domínio público (http://docs.google.com), criando assim, um questionário com endereço on-line que continham as instruções básicas, objetivos resumidos e informações sobre a natureza da pesquisa. Com o link gerado, foi solicitada a participação voluntária dos internautas através de redes sociais. Os participantes também foram informados que ao responder o formulário, automaticamente estariam concordando em participar da pesquisa, e que sua participação no estudo não traria qualquer prejuízo. Depois de respondidos os questionários, as respostas eram enviadas, automaticamente, para um banco de dados com acesso exclusivo dos pesquisadores envolvidos.

\section{Análise dos Dados}

Os dados foram analisados através do pacote estatístico PASW (Predictive Analytics SoftWare) versão 18. Por meio deste, realizaram-se as estatísticas descritivas (medidas de tendência central e distribuição de frequência), o teste $\mathrm{t}$ - Student para verificar o poder discriminativo dos itens, a Análise dos Componentes Principais (PCA), foi usada para conhecer a estrutura da escala, enquanto que se utilizou da análise de regressão para verificar validade preditiva do instrumento. E em seguida adotado o índice de consistência interna alfa de Cronbach $(\alpha)$ e homogeneidade (correlação item-item) para verificar a fidedignidade da CIUS.

\section{Resultados}


Aqui os resultados são descritos em subseções, de acordo com as análises estatísticas que foram usadas e na sequência em que foram realizadas; sendo primeiro o poder discriminativo dos itens, seguido respectivamente pela validade de construto, validade preditiva e consistência interna.

\section{Poder Discriminativo dos Itens}

Com o intuito de constatar se os itens conseguem discriminar sujeitos com pontuações próximas, fez-se uso desta análise usando a mediana empírica para definir os grupos-critério internos (Pasquali, 2013). Para tanto, foi utilizada a pontuação total da escala (soma de todos os itens do instrumento), posteriormente esta foi classificada em grupos inferiores e superiores tendo em conta a posição relativa à mediana. Em seguida, os itens foram testados através do teste $t$ - Student para amostras independentes, sendo considerado satisfatório, o poder de discriminação, de itens que conseguiram diferenciar os grupos. Os resultados estão sumarizados na Tabela 1.

Tabela 1.

Poder discriminativo dos itens

\begin{tabular}{|c|c|c|c|c|c|c|}
\hline \multirow{3}{*}{ Itens } & \multicolumn{4}{|c|}{ Grupos-Critério } & \multirow{2}{*}{\multicolumn{2}{|c|}{ Contraste }} \\
\hline & \multicolumn{2}{|c|}{ Inferior } & \multicolumn{2}{|c|}{ Superior } & & \\
\hline & $\mathrm{M}$ & DP & $\mathrm{M}$ & DP & $t(187)$ & $p$ \\
\hline 01. & 1,37 & 0,950 & 2,44 & 0,975 & $-7,598$ & 0,001 \\
\hline 02. & 1,16 & 0,860 & 2,56 & 0,886 & $-10,982$ & 0,001 \\
\hline 03. & 0,58 & 0,920 & 2,30 & 1,333 & $-10,345$ & 0,001 \\
\hline 04. & 0,40 & 0,728 & 1,56 & 1,131 & $-8,481$ & 0,001 \\
\hline 05. & 0,86 & 0,950 & 2,43 & 1,260 & $-9,725$ & 0,001 \\
\hline 06. & 0,74 & 0,854 & 2,24 & 1,056 & $-10,833$ & 0,001 \\
\hline 07. & 0,55 & 0,671 & 2,05 & 1,143 & $-11,106$ & 0,001 \\
\hline 08. & 1,04 & 0,999 & 2,37 & 0,946 & $-9,335$ & 0,001 \\
\hline 09 . & 0,52 & 0,886 & 1,86 & 1,158 & $-8,975$ & 0,001 \\
\hline 10. & 0,55 & 0,749 & 2,14 & 1,140 & $-11,433$ & 0,001 \\
\hline 11. & 0,38 & 0,628 & 1,55 & 1,159 & $-8,684$ & 0,001 \\
\hline 12. & 1,38 & 0,986 & 2,94 & 1,013 & $-10,671$ & 0,001 \\
\hline 13. & 0,80 & 0,922 & 2,43 & 1,244 & $-10,263$ & 0,001 \\
\hline 14. & 0,71 & 0,820 & 2,37 & 1,143 & $-11,481$ & 0,001 \\
\hline
\end{tabular}


Como podem ser observados, na Tabela 1, os resultados do teste $t$ indicam que todos os itens discriminam satisfatoriamente $(t \geq 1,92 ; p<0,05)$. Portanto, conseguem diferenciar os indivíduos com pontuações altas e baixas na escala. Essa característica assegura a qualidade métrica individual dos itens. Com esse aspecto assegurado passa-se para a próxima etapa que objetiva verificar a validade do instrumento e sua consistência interna.

\section{Validade de Construto}

Para verificar a validade fatorial (validade de construto) da CIUS, ou seja, se a adequação da definição do construto que essa escala mede, reflete realmente o verdadeiro significado teórico do mesmo, foi usada a Análise dos Componentes Principais (ACP). Primeiramente, buscou-se averiguar a fatorabilidade da matriz utilizando do KaiserMeyer-Olkin (KMO), que é um índice que trabalha com as correlações parciais das variáveis, onde são aceitos valores iguais ou superiores a 0,60; e o Teste de Esfericidade de Bartlett que comprova a hipótese de que a matriz de covariâncias é uma matriz de identidade. Tal hipótese é rejeitada quando os valores são significativos. Os resultados encontrados confirmam a possibilidade de ser realizada uma ACP $[\mathrm{KMO}=0,91$ e Teste de Esfericidade de Bartlett, $\left.\chi^{2}(91)=1373,697 ; p<0,001\right]$.

Superada esta etapa, procedeu-se a ACP, sem fixar rotação ou número fixo de dimensões a serem extraídas, com intuito de se conhecer a estrutura do instrumento. Ao observar os valores próprios (eigenvalues), ou critério de Kaiser, percebe-se a existência de evidências que apontam a existência de dois componentes (eigenvalues $\geq 1$ ). No entanto, ao se atentar ao scree test (critério de Cattell), nota-se que apenas um dos valores próprios se destaca aos demais, como pode ser visto na Figura 1.

Figura 1.

Representação Gráfica dos Valores Próprios 


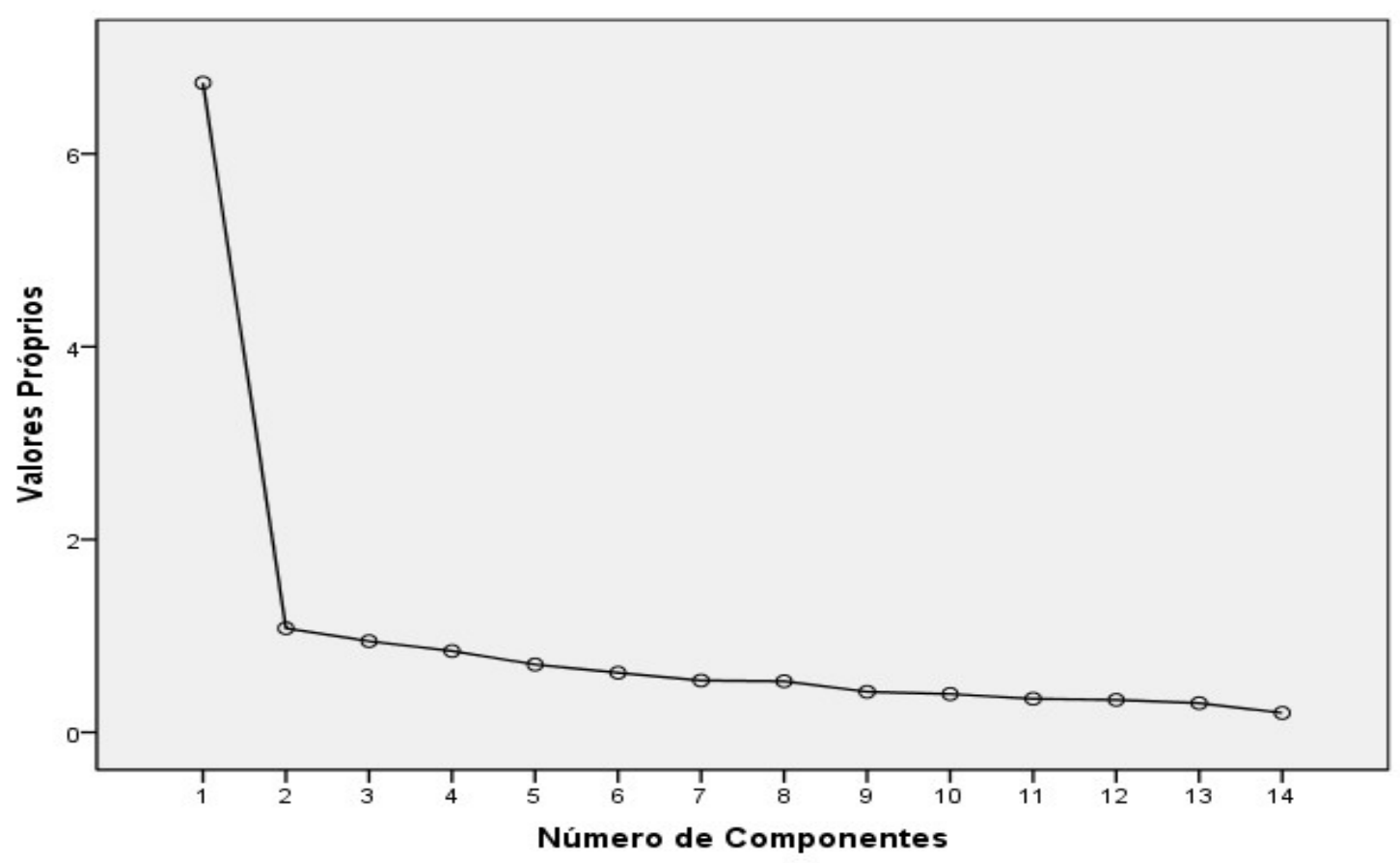

Fica evidente, na figura, que apenas um dos valores próprios se discrepa em relação aos outros, indicando, portanto, a extração de apenas um fator. No entanto, para dirimir eventuais dúvidas, quanto ao número de fatores, realizou-se uma Análise Paralela que é usada para comparar os valores próprios empíricos com aqueles 1.000 obtidos paralelamente por meio de matrizes randômicas com características semelhantes ao banco de dados empírico (número de itens e tamanho amostral). Essa análise propõe que os dados empíricos devem explicar mais variância que aqueles gerados aleatoriamente. Ao comparar os valores próprios, caso o valor próprio observado, correspondente, for maior que o simulado, apoia a existência do fator. Seguindo esta orientação, observou-se que somente um valor próprio (o mais alto) foi superior ao seu correspondente simulado, apoiando assim a existência de um único componente.

De posse de tais informações, rodou-se uma segunda ACP, agora fixando a extração de um componente, e levando em consideração o escore fatorial mínimo de 0,30 (Pasquali, 2016). Como se pode perceber na Tabela 2, todos os itens saturaram de maneira satisfatória no componente geral denominado uso compulsivo da internet.

Tabela 2.

Estrutura Fatorial da Escala de Uso Compulsivo da Internet (CIUS) 


\begin{tabular}{clc}
\hline 07 & Você costuma pensar na sua próxima sessão na internet? & 0,77 \\
06 & Você pensa na internet, mesmo quando não está online? & 0,75 \\
14 & Você se sente inquieto, frustrado ou irritado quando você não pode & 0,75 \\
& usar a Internet? & 0,75 \\
10 & Você apressa seus trabalhos (em casa), a fim de entrar na internet? & 0,71 \\
02 & Você continua a usar a Internet, apesar de sua intenção de parar? & 0,71 \\
03 & Os outros (por exemplo, cônjuge, filhos, pais) dizem que você deve & 0,71 \\
& usar a Internet menos? & 0,70 \\
12 & Você acessa a internet quando está se sentindo para baixo? & 0,68 \\
13 & Você usa a Internet para escapar de seus sofrimentos ou obter alívio & 0,66 \\
& de sentimentos negativos? & 0,66 \\
05 & Você perde sono por causa da Internet? & 0,65 \\
01 & Você acha difícil parar de usar a Internet quando você está online? & 0,64 \\
09 & Você já tentou sem sucesso passar menos tempo na internet? \\
08 & Você acha que deveria usar a Internet com menos frequência? & 0,60 \\
11 & Você negligência as suas obrigações diárias (trabalho, escola ou & \\
& família), porque você prefere acessar a Internet? & 0,59 \\
04 & Você prefere usar a Internet em vez de gastar tempo com as outras & 14 \\
& pessoas (por exemplo, cônjuge, filhos, pais)? & 6,73 \\
& Número de itens & 48,1 \\
& Valor Próprio & 0,91 \\
\hline & \%o da variância \\
& alfa de Cronbach
\end{tabular}

Assim como no estudo original, o instrumento apresentou-se unidimensional com valor próprio de 6,73 , explicando aproximadamente metade da variância total $(48,1 \%)$ e apresentando itens com cargas fatoriais variando de 0,59 a 0,77 .

\section{Validade Preditiva}

Adicionalmente, apesar de não ser o objetivo final deste estudo, buscou-se verificar sua validade preditiva (validade de critério). Esse parâmetro refere-se ao grau de eficácia que um teste tem em predizer um desempenho específico (Pasquali, 2016), neste caso, verificar se o grau em que as pessoas usam a internet pode predizer a quantidade de horas que as mesmas passam online.

Para isso se utilizou de uma pergunta que foi colocada junto ao questionário sócio demográfico, que tratava da quantidade de horas diária dispensadas para uso da internet. Esta era respondida em uma escala de 8 pontos que variava de 0 (menos de uma hora) a 7 (sete horas ou mais). Para analisar, os dados encontrados, valeu-se de uma análise de regressão linear bivariada, os resultados demonstram que $20 \%\left(\mathrm{R}^{2}=0,20\right)$ da variância na resposta sobre a quantidade de horas online podem ser preditas pela pontuação total 
obtida na CIUS $[\mathrm{F}(1,199)=49,893, p<0,001)]$. Dessa forma, quanto maior for à pontuação de um sujeito na CIUS, maior será o tempo que ele gasta usando a internet ( $\beta$ $=0,45 ; \mathrm{t}=7,063, p<0,001)$.

\section{Consistência Interna}

Por fim, para verificar a consistência interna, como indicador de precisão, valeuse do coeficiente alfa de Cronbach $(\alpha)$. Como esperado, a CIUS apresentou consistência interna $(\alpha=0,91)$ considerada satisfatória, além de índice de homogeneidade (correlação item-item - $r_{\text {iii }}$ ) de 0,44, também meritória.

\section{Discussão}

O presente estudo teve como principal objetivo a adaptação de uma medida para mensurar o uso compulsivo de internet para Brasil, a Escala de Uso Compulsivo da Internet (CIUS; Meerkerk et al., 2009), tendo em conta a escassez de medidas que busquem conhecer o uso compulsivo da internet. Acredita-se que esse objetivo tenha sido alcançado, a CIUS, assim como na sua elaboração, no contexto brasileiro se mostrou unidimensional, explicando quase metade da variância total. A versão final, adaptada para o Brasil, composta por seus 14 itens, é um instrumento curto, assegurando um dos prérequisitos básicos para um instrumento psicométrico, a parcimônia, podendo assim, explicar o máximo de informação com poucas variáveis (Pasquali, 2016).

A qualidade métrica dos itens foi averiguada e garantida a partir do poder discriminativo dos mesmos. Sem exceção, os itens apresentaram meritória capacidade de diferenciar escores altos e baixos ( $\mathrm{t}>1,96$ e $\mathrm{p}<0,001$; Pasquali, 2016), confirmando assim a possibilidade desses itens poderem diferenciar pessoas com pontuações próximas na CIUS.

Seus parâmetros psicométricos estão de acordo com as especificações recomendadas nas principais obras da literatura (Marôco, 2016), a fidedignidade do componente teorizado está dentro da indicada para uso em diagnóstico, inclusive superior $(\alpha>0,70)$, quesito respaldado pelo alto índice de homogeneidade (correlação item-item). Estes valores são, inclusive, melhores do que na oportunidade de sua elaboração $(\alpha=$ 0,89, $\alpha=0,89, \alpha=0,90$; Meerkerk et al., 2009) e similares àqueles encontrados em 
validações recentes, as quais apresentaram valores alfas entre 0, 90 e 0,99 (LopezFernandez et al., 2019).

A Análise dos Componentes Principais (PCA), considerando a ajuda da Análise Paralela permitiu averiguar que a CIUS é uma medida claramente unidimensional, avaliando comportamentos compulsivos de uso da internet. Ainda averiguou que este instrumento apresenta evidência de validade de critério, preditiva, ou seja, pode-se inferir que quanto maior o escore de um indivíduo maior será o tempo dispensado para uso da internet (Pasquali, 2016). Considerando o nível de precisão, no geral, observaram-se índices de consistência interna acima do recomendado na literatura, mesmo quando considerado todos os itens ao mesmo tempo (Hair, Anderson, Tatham, \& Black, 2018), neste sentido, justifica-se seu emprego em pesquisas futuras. Todavia, tal medida carece de maiores estudos, especialmente no que diz respeito à tentativa de verificar sua validade convergente.

No entanto, este, como qualquer outro trabalho científico, não está isento de limitações. Não há como não citar o viés da amostragem, pois esta não foi probabilística, mas tipo acidental (por conveniência), que compromete e não reflete a composição real da população, impossibilitando extrapolar os resultados aqui ora apresentados. Nem mesmo o número relativamente grande da amostra permite transpor tal limitação, no entanto, se faz importante apontar que o objetivo aqui não era o de se extrapolar os resultados encontrados, neste sentido, indica-se que em investigações futuras, utilizandose da CIUS, sejam consideradas amostras mais representativas e heterogêneas, coletadas de forma aleatória. Além disso, todos os questionários foram aplicados pela internet, o que pode ser um viés se levarmos em conta que indivíduos que usam a internet de forma compulsiva têm maior possibilidade de ser exposto a uma solicitação de participação de um estudo como o aqui reportado.

Mesmo com algumas restrições, em função das limitações repostadas, os resultados deste estudo são satisfatórios e plausíveis com a literatura consultada, seja em aspectos psicométricos ou acerca do uso compulsivo da internet. Logo, partindo das qualidades métricas aceitáveis (Marôco, 2016; Pasquali, 2016) aliadas à carência de instrumentos de medida para tal construto no Brasil, o presente estudo constitui-se como uma contribuição considerável. Entretanto, as limitações descritas anteriormente obriga, ao leitor, analisar com algumas ressalvas os resultados encontrados. 


\section{Considerações finais}

Por fim, sugere-se a utilização do instrumento aqui apresentado em pesquisas futuras, principalmente por ter tamanho relativamente curto e ser unidimensional e autoaplicável, sujeitos de diferentes faixas etárias podem ser avaliados, sendo útil para mapear e planejar estratégias de combate e prevenção do comportamento compulsivo em relação ao uso da internet, ou ainda conhecer seus antecedentes e consequentes, já que este padrão comportamental relaciona-se com diversos construtos.

Em suma, apresentam-se evidências suficientes da adequação psicométrica da Escala de Uso Compulsivo da Internet (CIUS). Esta medida constitui-se, portanto, um instrumento breve, de fácil compreensão, com itens curtos, que pode ser usado adequadamente em estudos em que o interesse é conhecer os antecedentes e consequentes de comportamentos compulsivos de uso da internet.

\section{Agradecimentos}

O presente artigo contou com apoio do CNPq e CAPES, por meio de financiamento projeto de pesquisa do $\mathrm{CNPq}$ e bolsa de produtividade ao primeiro autor, além da CAPES por conceder bolsa de Doutorado ao segundo e sexto autores, e bolsa de pós doutorado ao quinto autor. Aproveitamos para demonstrar nossa gratidão a estas instituições.

\section{Declaração de conflitos de interesse}

Os autores declararam não haver potenciais conflitos de interesse relacionados ao presente estudo.

\section{Referências}

Abreu, C. N., Karam, R. G., Góes, D. S., \& Spritzer, D. T. (2008). Dependência de internet e de jogos eletrônicos: uma revisão. Revista Brasileira de Psiquiatria, 30(2), 156-167. doi: 10.1590/S1516-44462008000200014 
American Psychological Association (1994) Diagnostic and statistical manual of mental disorders. (4th ed). Washington, DC: American Psychiatric Publishing.

Anderson, C. A., \& Bushman, B. J. (2018). Media Violence and the General Aggression Model. Journal of Social Issues, 74(2), 386-413. doi: 10.1111/josi.12275

Bean, A. M., Nielsen, R. K. L., van Rooij, A. J., \& Ferguson, C. J. (2017). Video game addiction: The push to pathologize video games. Professional Psychology: Research and Practice, 48(5), 378-389. doi: 10.1037/pro0000150

Deleuze, J., Long, J., Liu, T. Q., Maurage, P., \& Billieux, J. (2018). Passion or addiction? Correlates of healthy versus problematic use of videogames in a sample of Frenchspeaking regular players. Addictive Behaviors, 82, 114-121. doi: 10.1016/j.addbeh.2018.02.031

Ebeling-Witte, S., Frank, M. L., \& Lester, D. (2007). Shyness, internet use and personality. Cyberpsychology \& Behavior, 10(5), 713-716. doi: $10.1089 / \mathrm{cpb} .2007 .9964$

Griffiths, M. D. (1997). Exercise addiction: A case study. Addiction Research, 5(2), 161168.

Hair, J. F., Anderson, R. E., Tatham, R. L., \& Black, W. C. (2018). Multivariate Data Analysis. (8thed). Cengage, U.K.

Kerkhof, P., Finkenauer, C., \& Muusses, L. D. (2011). Relational Consequences of Compulsive Internet Use: A Longitudinal Study Among Newlyweds. Human Communication Research, 37(2), 147-173. doi: 10.1111/j.1468-2958.2010.01397.x

Lopez-Fernandez, O., Griffiths, M. D., Kuss, D. J., Dawes, C., Pontes, H. M., Justice, L., ... \& Männikkö, N. (2019). Cross-cultural validation of the Compulsive Internet Use Scale in four forms and eight languages. Cyberpsychology, Behavior, and Social Networking, 22(7), 451-464. doi: 10.1089/cyber.2018.0731

Mak, K. K., Nam, J. K., Kim, D., Aum, N., Choi, J. S., Cheng, C., ... \& Watanabe, H. (2017). Cross-cultural adaptation and psychometric properties of the Korean Scale for Internet Addiction (K-Scale) in Japanese high school students. Psychiatry Research, 249, 343-348. Doi: 10.1016/j.psychres.2017.01.044

Marôco, J. (2014). Análise de equações estruturais: fundamentos teóricos, software e aplicações (2.ed.). Pêro Pinheiro, Portugal: Report Number.

Meerkerk, G. J., Van Den Eijnden, R. J. J. M., Vermulst, A. A., \& Garretsen, H. F. L. 
(2009). The Compulsive Internet Use Scale (CIUS): Some Psychometric Properties. CyberPsychology \& Behavior, 12(1), 1-6. doi: 10.1089/cpb.2008.0181

Meerkerk, G. J., Van Den Eijnden, R. J. J. M., Franken, I. H. A., \& Garretsen, H. F. L. (2010). Is compulsive internet use related to sensitivity to reward and punishment, and impulsivity? Computers in Human Behavior, 26(4), 729-735. doi: 10.1016/j.chb.2010.01.009

Mottram, A. J., \& Fleming, M. J. (2009). Extraversion, impulsivity, and online group membership as predictors of problematic Internet use. Cyberpsychology \& Behavior, 12, 319-21. doi: 10.1089/cpb.2007.0170

Pasquali, L. (2016). TEP - Técnicas de Exame Psicológico: Os Fundamentos. (2 ed). São Paulo, SP: Vetor editora.

Penteado, C. L., Santos, M. B., Araújo, R. A., \& Silva, S. J. (2011). Ação política na internet brasileira. Perspectivas em ciência da informação, 16 (1), 111-132. Doi: $10.1590 /$ S1413-99362011000100007

Pontes, H. M. (2017). Investigating the differential effects of social networking site addiction and Internet gaming disorder on psychological health. Journal of Behavioral Addictions, 6(4), 601-610. doi: 10.1556/2006.6.2017.075

Rosenkranz, T., Müller, K. W., Dreier, M., Beutel, M. E., \& Wölfling, K. (2017). Addictive potential of internet applications and differential correlates of problematic use in internet gamers versus generalized internet users in a representative sample of adolescents. European addiction research, 23(3), 148-156. doi: 10.1159/000475984

Sebastian, W., Helen, C. W., Catherine, H. G., Karsten, D. W., Alexander, T. V., \& Marianne, J. (2018). Correlates of Mono- and Dual-Victims of Cybergrooming and Cyberbullying: Evidence from Four Countries. Cyberpsychology, Behavior, and Social Networking, 21(2), 91-98. doi: 10.1089/cyber.2016.0733

Studer, J., Marmet, S., Wicki, M., \& Gmel, G. (2019). Cybersex use and problematic cybersex use among young Swiss men: Associations with sociodemographic, sexual, and psychological factors. Journal of Behavioral Addictions, 8(4), 794-803. doi: $10.1556 / 2006.8 .2019 .69$

Van Zalk, N. (2016). Social anxiety moderates the links between excessive chatting and compulsive Internet use. Cyberpsychology: Journal of Psychosocial Research on Cyberspace, 10(3). doi: 10.5817/CP2016-3-3 
Wartberg, L., Kriston, L., Bröning, S., Kegel, K., \& Thomasius, R. (2017). Adolescent problematic internet use: Is a parental rating suitable to estimate prevalence and identify familial correlates?. Computers in Human Behavior, 67, 233-239. doi: 10.1016/j.chb.2016.10.029 